\title{
WCCC 2018: the 24th World Computer Chess Championship
}

Article

Accepted Version

The WCCC 2018 report

Krabbenbos, J., van den Herik, J. and Haworth, G. (2018)

WCCC 2018: the 24th World Computer Chess Championship.

ICGA Journal, 40 (3). pp. 182-193. ISSN 1389-6911 doi:

https://doi.org/10.3233/ICG-190080 Available at

https://centaur.reading.ac.uk/78436/

It is advisable to refer to the publisher's version if you intend to cite from the work. See Guidance on citing.

Published version at: https://doi.org/10.3233//CG-190080

To link to this article DOI: http://dx.doi.org/10.3233/ICG-190080

Publisher: The International Computer Games Association

All outputs in CentAUR are protected by Intellectual Property Rights law, including copyright law. Copyright and IPR is retained by the creators or other copyright holders. Terms and conditions for use of this material are defined in the End User Agreement.

\section{www.reading.ac.uk/centaur}

\section{CentAUR}

Central Archive at the University of Reading

Reading's research outputs online 


\section{WCCC 2018: The 24 ${ }^{\text {th }}$ World Computer Chess Championship}

Jan Krabbenbos, Jaap van den Herik and Guy Haworth ${ }^{1}$

Amersfoort, the Netherlands, Leiden, the Netherlands and Reading, UK

The $24^{\text {th }}$ World Computer Chess Championship started on July 16, 2018. Eight programs as in Table 1 participated in a round robin tournament of seven rounds. The tournament took place at the Stockholmsmässan in Stockholm, Sweden and was organised by the ICGA. The venue was part of the international conferences IJCAI, ECAI, AAMAS, ICML, ICCBR and SoCS. The main sponsor was Digital Game Technology, DGT. It was held in conjunction with the 8th World Computer Software Championship and the 2018 Computer Speed Chess Championship. The classical tempo was $1 \mathrm{~h} 45^{\prime}+15^{\prime \prime} /$ move for each side.

Table 1

The participants in WCCC 2018 (CPW, 2018)

\begin{tabular}{|c|c|c|c|c|c|c|c|c|}
\hline$\pi$ & Engine & Author(s) & State & Operator & Platform & Cores & Opening Book & EGTs \\
\hline 1 & BOOOT & Alex Morozov & UA & A M & $2 \times$ Xeon & 28 & Arena Perfect, abs & - \\
\hline 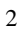 & CHIRON & Ubaldo Andrea Farina & IT & U A F & 2 x AMD EPYC 7551 & 64 & Private + GOI (poly glot) & $6 \mathrm{~m}$ Syzygy \\
\hline 3 & GRIDGINKGO & $\begin{array}{c}\text { Frank Schneider, Kai } \\
\text { Himstedt and Rob Hyatt }\end{array}$ & DE & $\begin{array}{l}\text { K H and Timo } \\
\text { Haupt }\end{array}$ & 40 x Intel Xeon E5-2630 v3 & 320 & Wolfgang Zugrav & 6m Nalimov \\
\hline 4 & JONNY & Johannes Zwanzger & DE & $\mathrm{J} \mathrm{Z}$ & $108 \times$ AMD Opteron & 1296 & Own & $6 \mathrm{~m}$ Syzy gy \\
\hline 5 & KOMODO & $\begin{array}{c}\text { Don Dailey, Mark Lefler, } \\
\text { and Larry Kaufman }\end{array}$ & US & Erdogen Günes & 4 x Intel Xeon E7-8890 v2.2 & 58 & Erdogan Günes & $\begin{array}{l}\text { 6-man } \\
\text { Syzygy }\end{array}$ \\
\hline 7 & SHREDDER & Stefan Meyer-Kahlen & $\mathrm{DE}$ & S M-K & Intel Xeon & & Own, small, handmade & Syz \& Shredder \\
\hline & THE BARON & Richard Pijl & NL & $\mathrm{R} \mathrm{P}$ & $2 \times 14$-core Xeon & 28 & Own & $6 \mathrm{~m}$ Syzy gy \\
\hline
\end{tabular}

This year the ICGA revived the tradition of a chess grandmaster explaining the games and entertaining the public. Evolution since the 1986 WCCC in Cologne has been considerable. In 1986 grandmaster Vlastimil Hort gave comments from the point of view of a chess player who knew better than the computer. In this tournament, the Swedish commentator Harry Schussler GM knew that almost all programs were stronger than he was. Nevertheless he did a very good job of pointing out which moves were typical for a computer and still very strong moves. He remarked that humans could learn a substantial amount from the computer manoeuvres.

Round 1: LEELA CHESS ZERO - KOMODO 0-1, BOOOT - JONNY 1/2-1/2, GRIDGINKGO - THE BARON $1-0$, CHIRON - SHREDDER $1 / 2-1 / 2$

In the first round, LEELA CHESS ZERO and KOMODO played an exciting game that only failed to end in a draw because White overstepped the clock. In the opening, the key feature was the isolated central black pawn on $\mathrm{d} 5$. Was it a strong asset in the centre? Yes, but White, in taking the pawn would have

\footnotetext{
${ }^{1}$ Corresponding author: g.haworth@ reading.ac.uk
} 
given Black even more chances. At the first opportunity, White preferred to castle kingside and not to take the pawn: indeed that was impossible. Only a few moves later small combinations played a key role in the game's complexities. Black decided to play 15 .... Na2 , a very remarkable move, see Fig. 1a.

The continuation was 15. ... Na2 16. Red1 Qxb3 17. Bxd5 Rxd5 18. Qxd5 Qxd5 19. Rxd5 Be6 20. Rd2 resulting in an early endgame in which Black had two pieces for a rook and a pawn. The final phase, sometimes exciting and at other times rather boring, lasted more than 110 moves. At that time, the tablebases and human chess players with even mediocre knowledge of the game all knew the game was drawn. Even so, Black continued to play since White did not have the use of endgame tablebases and had to compute all variations. This took a lot of time and on move 134, White overstepped the time limit and sadly forfeited the draw.
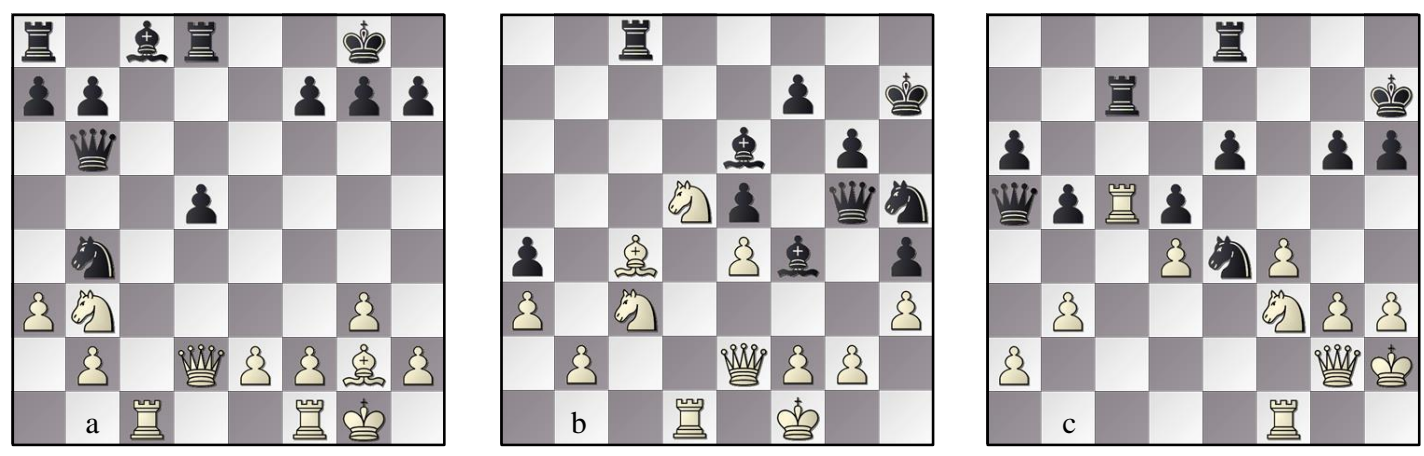

Fig. 1. R1: a) LeEla Chess Zero - KOMOdo 15b; b) BoOot - JonNy 37b; c) GRIDGinKGO - The BARON 34w.

The game BOOOT-JONNY started as a quiet Semi-Slav. Black had a plan to attack the white king and for the execution of the plan JONNY transferred all its pieces to the kingside. BOOOT reacted adequately and gave Black the impression that the square $\mathrm{f} 4$ was overloaded since it should be used for the bishop as well as for the knight. For human beings this difficulty would be a challenging task to resolve but this is not so for computers of current playing strength. On move 37 as in Fig. 1b, Black played 37... Bh2 in order to be able to play 38... Nf4.

To the end, the black bishop remained on h2. The black attack was vigorous but White was very alert in its defence, meanwhile creating counterplay in such a way that the game ended in repetitions of position. After a hard fight, this was a well-deserved draw for both sides.

The second program that decided a game to its advantage was GRIDGINKGO. It won by accurate play and by taking very fine decisions. The main point was that it sacrificed the exchange for a passed pawn. It occurred in an attractive series of moves that started with 34. b4, see Fig. 1c.

The continuation was 34. ... Qb6 35. Qc2 Nxc5 36. dxc5. Black then decided to create space, sacrificing the e-pawn by playing 36. ... e5. It was not clear if better moves were available.

In the course of the game GRIDGINKGO was able to challenge THE BARON further on the kingside and succeeded in creating a passed f-pawn. A nice interplay of king, rook and pawns led to the finale, a well-deserved win.

The game CHIRON - SHREDDER was a high-level game between opponents who had respect for the playing strength of the other. After a well-known opening, they soon followed their own paths. The 
strategic lines that were followed were understandable for human beings. No explanatory artificial intelligence was necessary to understand what motives were underlying the moves. The game was clear up to move 32 where Black decided to achieve a draw in the most decisive way although it was reached by a slightly surprising sequence of moves.

Standings after round 1: 1-2 GridGinkGo, Komodo 1; 3-6 BoOOT, CHIRON, JonNY, SHREdDER 1/2; 7-8 LEELA CHESS ZERO, THE BARON 0

Round 2: KOMODO - SHREDDER 1/2-1/2, THE BARON - CHIRON 1/2-1/2, JONNY - GRIDGINKGO 1/2-1/2, LEELA CHESS ZERO - BOOOT $1 / 2-1 / 2$

The round resulted in four draws. One game, KOMODO - SHREDDER, was tough and inspiring, two games were very long and require some perseverance when being replayed, and the game between THE BARON and CHIRON ended in a draw without much excitement since they quietly exchanged all pieces.

In the game KOMODO - SHREDDER, the most interesting point was after move 18. ... axb6, see Fig. 2a, when White played 19. Nd6. The continuation was 19. ... Bxd6 20. Bxb7 Rxe1+ 21. Rxe1 Rd8 22. Rd1 Bc7 23. Rxd8 Bxd8. After 24. Kf1 White had two small advantages, namely the bishop pair against bishop and knight and the distant file-a/b pawns whereas Black had pawns on files b and $\mathrm{c}$. Nevertheless, the two advantages were insufficient for a win.

THE BARON - CHIRON has been discussed above: a straightforward draw. JONNY - GRIDGINKGO promised to be a sharp game after 13. h4 Bxh4 14. Qf3, see Fig. 2b. However, Black managed to hold on to equality and around move 30 the pawn positions were so interwoven that both sides took 130 moves to find an opening. It was all in vain and on move 164, a draw was agreed when repetition of position occurred on the board after $5 \frac{1}{2}$ hours of play.
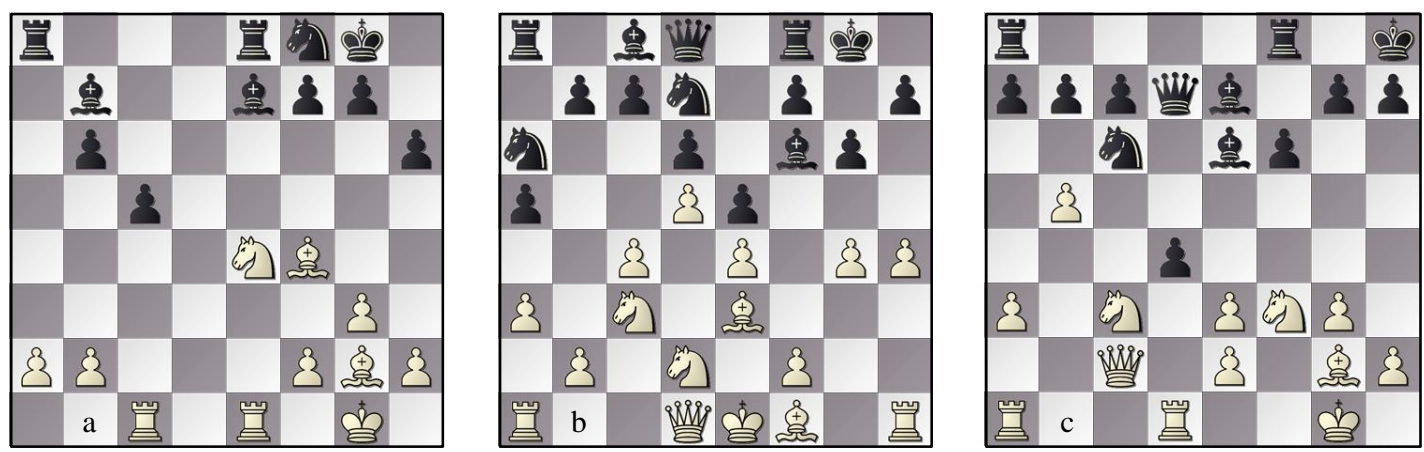

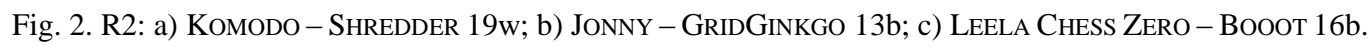

The game LEELA CHESS ZERO versus BOOOT was a game with one tense moment when White played 16. b5 as in Fig. 2c. After 16. ... Na5 17. Nxd4 the weighing of the pros and cons involved very different features. Nevertheless, there was no imbalance and hence the game ended in a draw.

Standings after round 2: 1-2 GridGINKGO, KOMOdo 11/2; 3-6 BOOOT, CHIRON, JonNy, SHREDDER 1; 7-8 LEELA CHESS ZERO, THE BARON $1 \frac{1}{2}$

Round 3: BOOOT - KOMODO 0-1, GRIDGINKGO - LEELA CHESS ZERO 1-0, CHIRON - JONNY 1/2-1/2, SHREDDER - THE BARON 1-0 
In this round, KOMODO played a very interesting game with BоOоT. It was a Berlin Wall opening: Black usually has a solid position but has to wait until the endgame before it is possible to have any advantage from the micro benefits collected to that point. Commentator and grandmaster Schussler made the statement that move 13. $\mathbf{f} 4$ is possibly the first advantage handed to Black, ceding the square f5 to the black pieces. What else could White have been played? In Harry's opinion, White should have sacrificed the e-pawn earlier than it did in the game.
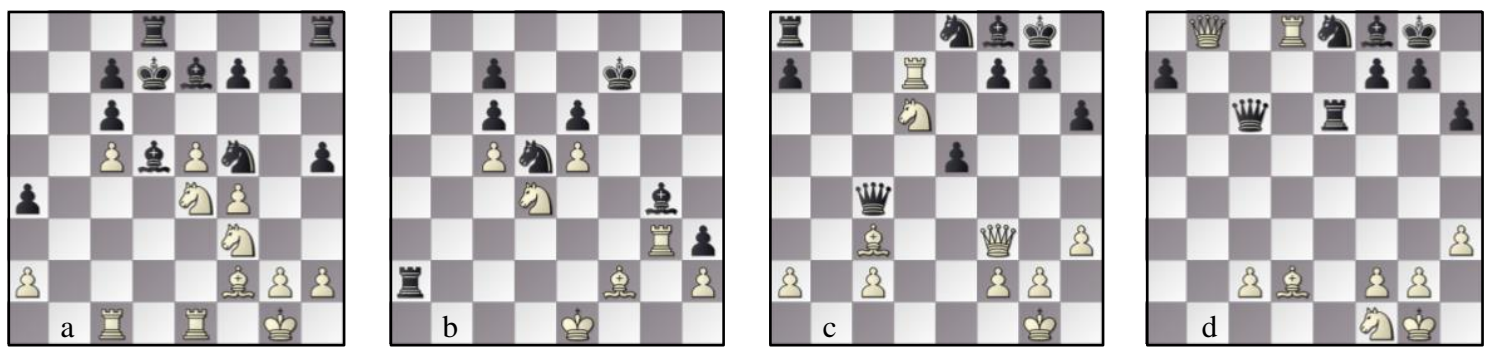

Fig. 3. R3: Booot - Komodo (a) 25w and (b) 78b; GridGinKGO - LeEla CHESS Zero (c) $29 \mathrm{~b}$ and (d) 39w.

On move 25 White played e6+, see Fig. 3a, sacrificing a pawn and obtaining some counterplay. In the sequel of the game White managed to regain the pawn but its position was difficult to defend. On move 78 as in Fig. 3b, Black played Nf4 and that was the start of its run to the win. 79. Rxg4 would have triggered the line $\mathrm{Nd} 3+80 . \mathrm{Kf} 1 \mathrm{Rxf} 2+81 . \mathrm{Kg} 1 \mathrm{Rb} 2$. White soon resigned.

The game GRIDGINKGO - LEELA CHESS ZERO had a difficult start. LEELA CHESS ZERO's operator was unfortunately beset by a family accident that put his mother in hospital. Another operator had to be called in which took some time but all participants were happy to accommodate and resolve the situation. Both opponents agreed to play the game under a time control giving GRIDGINKGO one hour and LEELA CHESS ZERO 45'+15"/move. The start of the game showed that both players did not intend to make it a quiet game. After a turbulent opening with Black taking the pawn on b2, White started complications with 29. Nd6 and the threat to capture the rook on a8 as in Fig. 3c.
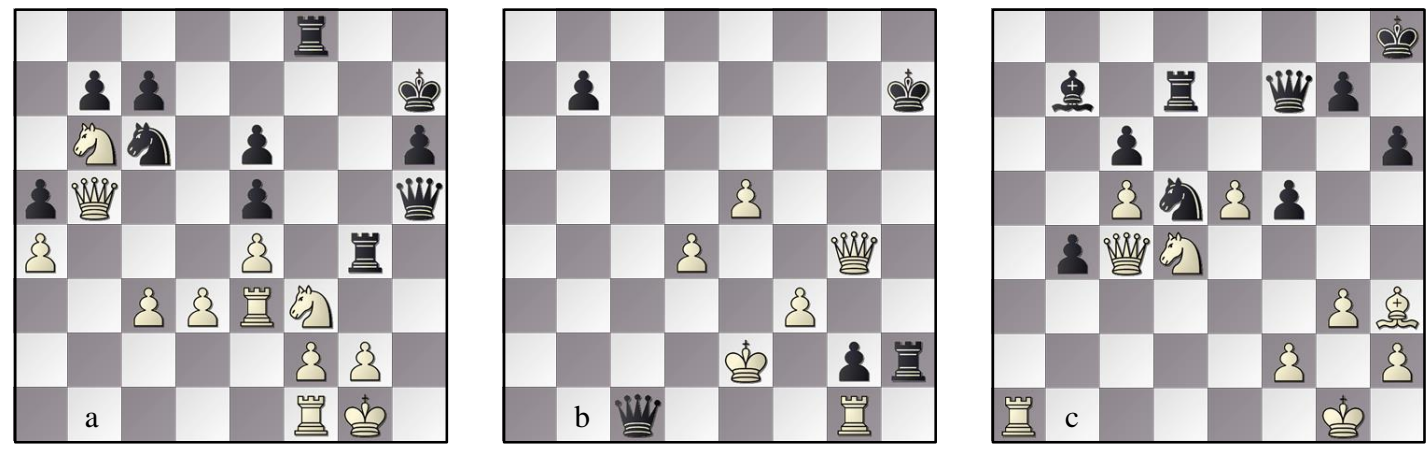

Fig. 4. R3: CHIRON - JonNy (a) 44b and (b) 76w; c) SHREDDER - THE BARON 38b.

The continuation was 29. ... e4 30. Nxe4 Qe6 31. Rd3. White subsequently activated with 39. g4 as in Fig. 3d. Commentator Harry Schussler GM mentioned that a human grandmaster would not immediately consider this move as a good one. Whatever the case, White succeeded in increasing its advantage and won the game convincingly. 
The game CHIRON - JONNY was quite exciting although it took a long time before real action made the game attractive. After 44. Nxb6, see Fig. 4a, Black played 44. ... Rfg8 which was followed by 45. Ne1 Rh4 46. f3 cxb6. Another exciting moment was after 69.... Qg3+ when White triggered the line 70. Kf1 h3 71. Ng4 hxg2+ 72 Ke2 Qf4 73 Qb6 Rxg4 74 Qe6+ Kh7 75 Qxg4 Qc1, which deserves Fig. 4b. With such trouvailles, all chess aficionados will agree that such a game should end in a draw as happened on move 84 .

In SHREDDER - THE BARON, Black relied on the well-known Dutch opening. It is a sharp opening in which both programs must know the opponent's opportunities very well in order to hold their own. After the opening White appeared to have manoeuvred better than Black although the situation was complex and Black had many resources. For a human being they are difficult to find but for a computer it is only a matter of deep look ahead. For instance, 38... Nf4, see Fig. 4c. 39. e6 Nxh3+ 40. Kh1 Qg8 41. exd7 Qxc4 42. d8Q+ Kh7 43. Qd7 Qxc5 44. Qxg7+ Kxg7 45. Ne6+ Kf6 46. Nxc5. THE BARON did not manage to reduce the pressure on its position. SHREDDER handled the game in a really grandmaster way and forced a win although it took 66 moves.

Standings after Round 3: 1-2 GRIDGINKGO, KOMODO 21/2; 3 SHREDDER 2; 4-5 CHIRON, JONNY 11/2; 6 BOOOT 1; 7-8 LEELA CHESS ZERO, THE BARON $1 / 2$

Round 4: KOMODO - THE BARON 1-0, JONNY - SHREDDER 1/2-1/2, LEELA CHESS ZERO - CHIRON $1 / 2-1 / 2$, BOOOT - GRIDGINKGO 1/2-1/2

The game KOMODO - THE BARON was a typical game in which good preparation turned out to be effective. KOMODO went for an opening variation in which White offers a pawn for good play. The regain of the pawn was not obvious but the freedom of movement and the opportunity to create pressure was more than sufficient compensation for the pawn. Although THE BARON was ingenious and had many nice defences, the fact that it had not castled to the kingside turned out to be disastrous. White took a long time but was able to materialize the advantage. A win for KOMODO.

The game JONNY - SHREDDER was a game between old friends who are also old opponents. The programmers know each other very well and in this time of AI development, we may state that the programs know what the intentions are of the opposing program. In the game, this was expressed in a well-known opening followed by playing a long-trodden path without any excitement, which implied that the game ended on move 51 in a draw.
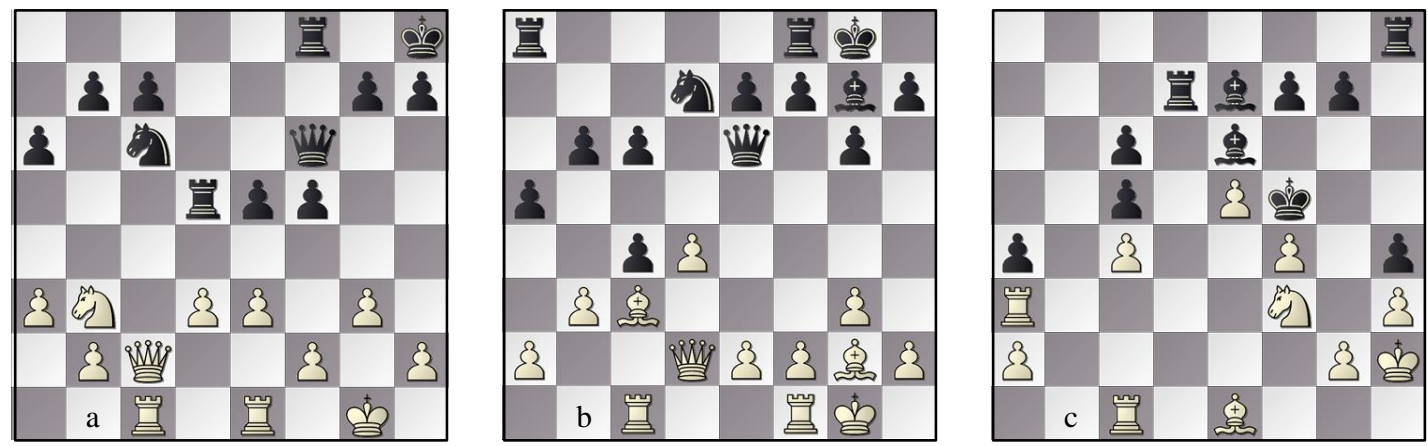

Fig. 5. a) R4, LEELA CHESS ZERO - CHIRON 22b; b) R4, BOOOT - GRIDGinKGo 17b;

c) R5, GRIDGINKGO - KOMODO 40w. 
LEELA CHESS ZERO - CHIRON also ended in a draw but this happened after a tough fight in which both sides attempted to take the initiative. At the strong level that programs nowadays have, it is possible to anticipate the ideas of the opponent to a large extent. On its 22nd move Black as in Fig. 5a started a small and neat combination that ended in a perpetual check opportunity: 22 .... f4 23 . Nc5 fxe3 24. fxe3 Rxc5 25. Qxc5 Qf2+ 26. Kh1.

The game BOOOT - GRIDGINKGO ended also in a draw but here it happened after some nice small combinations which did not change the equilibrium. From Fig. 5b, we show the main line which ended in a draw. Still, the variation had some unexpected twists. 17. ... cxb3 18. d5 Bxc3 19. Rxc3 Qd6 20. Rxc6 Qb4 21. Qxb4 axb4 22. axb3 with an equal endgame.

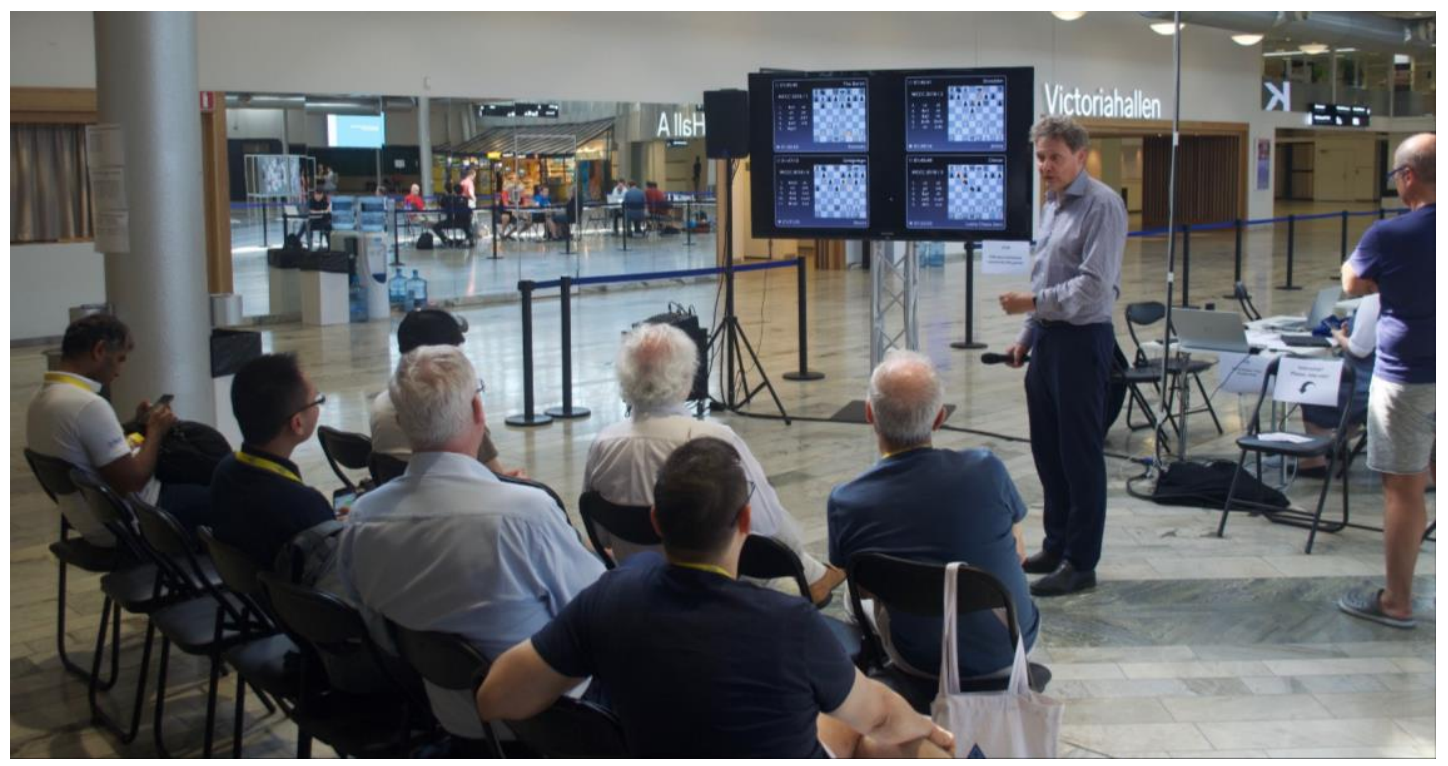

Fig. 6. GM Harry Schussler commentating on the opening choices in WCCC Round 4.

Standings after Round 4: 1 Komodo 31/2; 2 GRIDGINKGO 3; 3 SHREDdER 21/2; 4-5 CHIRON, JonNY 2; 6 BOOOT 11/2; 7 LEELA CHESS ZERO 1; 8 THE BARON $1 \frac{1}{2}$

Round 5: GRIDGINKGO - KOMODO $1 \frac{1}{2-1 / 2}$, CHIRON - BOOOT 1/2-1/2, SHREDDER - LEELA CHESS ZERO $1 / 2-1 / 2$, THE BARON - JONNY $1 / 2-1 / 2$

The game GRIDGINKGO - KOMODO started with a Berlin Defense. Games resulting from this opening are always tough games. They are dominated by small issues and small, very small advantages. It is the opening for games of world champion calibre. Even grandmasters have difficulties in evaluating the moves played. After some small challenges given by White to Black, we saw that both sides were open for a decisive exchange of pawns each on different sides of the board. See Fig. 5c after 39.... Rd7. At this moment, GRIDGINKGO judged that it was opportune to take with 40. Rxa4. Black replied with 40. ... Kxf4. There are many variations to be analysed as to why Black was unable to take the f4 pawn at an earlier stage. As stated above, to both programs this seemed to be the right moment. It also implied that both programs maintained the balance and this being so they found a nice variation that made it clear that the game would end in a draw. After 47... Kf5 we saw the position of Fig. 7a 
and line 48. Rxe7 Rxe7 49. Bxc5 Reb7 50. Nd4+ Kf4 51. Bxb4 Rxb4 52. Rf1+ Ke4 53. Nxc6 Rxc4 54. Nd8 Rc7 55. Nxe6 fxe6. After two more moves, a draw was agreed.

CHIRON - BOOOT ended in draw after a very exciting game. We highlight the excitement with two snapshots. The first one appeared after 19. f3, see Fig. 7b: 19. ... cxd4 20. cxd4 Bf6 21. fxe4 dxe4 22. h4 Rad8 23. Qa4 Qxd4+ 24. Qxd4 Rxd4 with strongly placed pawns in the center and a piece down for Black. The second snapshot is Fig. 7c after 30. Nc3: 30. ... Rxc3 31. bxc3 Bxc3 32. Ba3 Ra8 33. Be7 Bxa1 34. Rxa1 Rxa4 35. Rxa4 Bxa4 and seven more moves saw a draw agreed.
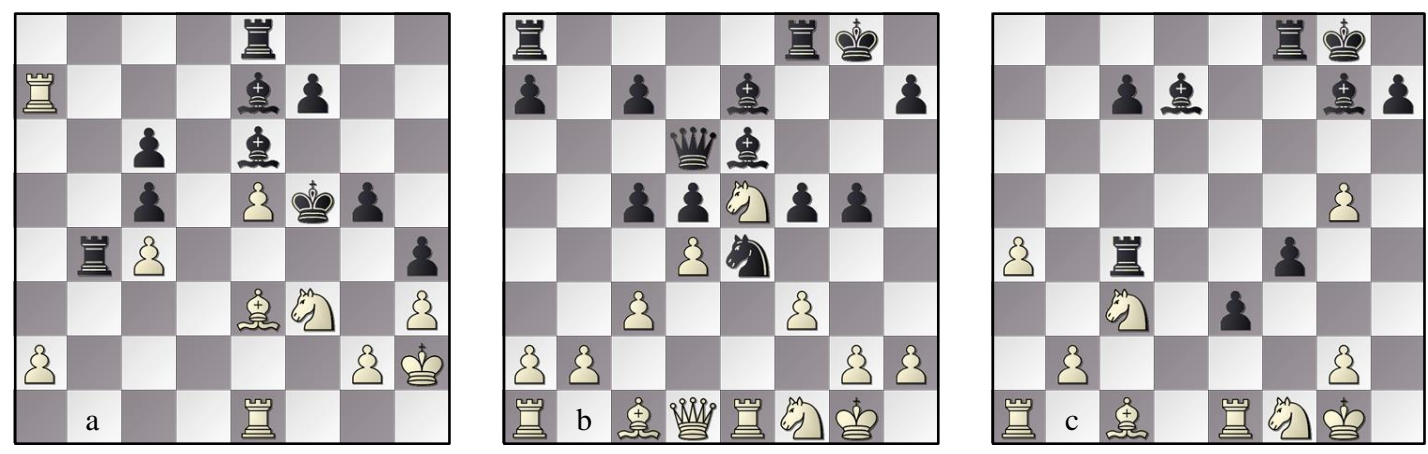

Fig. 7. R5: a) GRIDGINKGO - KOMOdO, 48w; CHIRON - BOOOT (b) $19 \mathrm{~b}$ and (c) $30 \mathrm{~b}$.

The game SHREDDER - LEELA CHESS ZERO was also an exciting game. They played mainly on their own strengths, which is to say without opening books. The positions on the board required utmost calculation. In the position of Fig. 8a, Black took a clear decision, effecting the transition to a smooth endgame and a draw which was well deserved by both sides. 48. ... N6xh5 49. Qd8 Nf6 50. Rb8 Ng6 51. Rf1 Rd6 52. Qc8 Qa2 53. Ra8 Qb3 54. Qf5 Qe3 55. Bg2 Re6 56. Rb8 Nh4 and after 57. Qf2 Nxg2 58. Kxg2 Qxe4+ 59. Qf3 Qxg4+ 60. Qxg4+ Nxg4, the draw followed.

THE BARON - JONNY was a quiet game in which the opponents may have been intent on an exciting game but this did not materialise. The game was one in which pieces harmoniously disappeared from the board and in the end on move 45 the material was reduced in such a way that a draw was the natural outcome.

Standings after Round 5: 1 KOMODO 4; 2 GRIDGINKGO 31/2; 3 SHREDDER 3; 4-5 CHIRON, JONNY 21/2; 6 BOOOT 2; 7 LEELA CHESS ZERO 11/2; 8 THE BARON 1
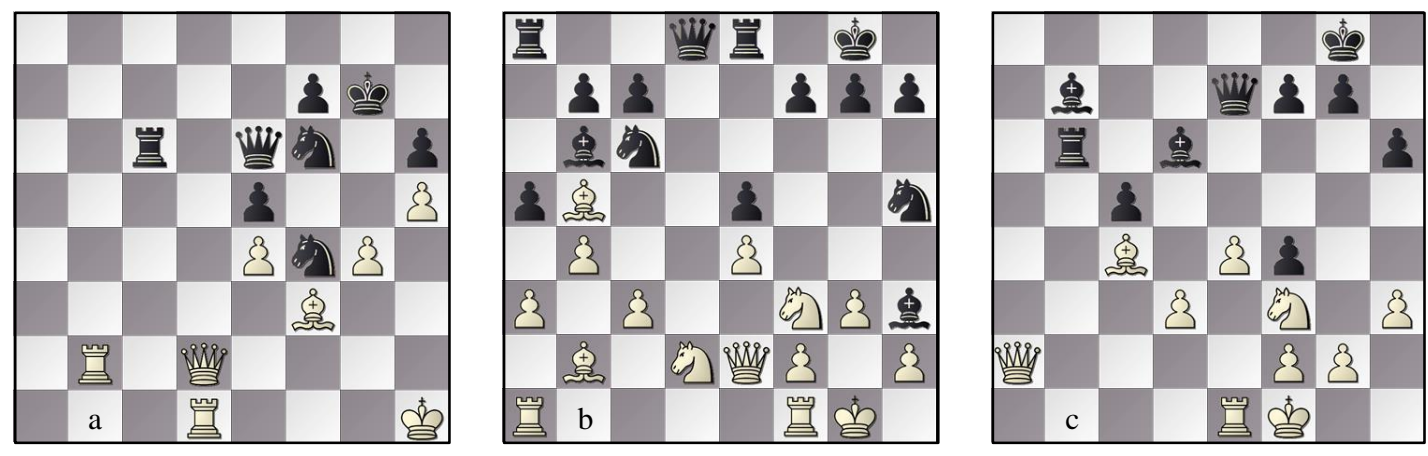

Fig. 8. a) R5, SHREDDER - LEELA CHESS ZeRo 48b; b) R6, KOMODO - JONNY 14w; c) R6, LEELA CHESS ZERO - THE BARON 37w. 
Round 6: KOMODO - JONNY 1/2-1/2, LEELA CHESS ZERO - THE BARON 1/2-1/2, BOOOT - SHREDDER $1 / 2-1 / 2$, GRIDGINKGO - CHIRON $1 / 2-1 / 2$

In the sixth round as in the fifth, all four games ended in a draw. Two were normal draws and two were exciting draws although the degree of excitement differed. It implied that there was no change to the engines' ranking. In the game KOMODO - JONNY the opening was the Berlin Defense in the Ruy Lopez opening. It was the closed variation and the programs followed their own ideas. After 13 moves the position of Fig. 8b appeared. Here KOMODO came up with a fascinating idea which clearly shows that current programs excel in estimating the intrinsic value of a position and thus also of a move that leads to this position. In this situation, it played 14. c4, with the idea of exchanging the bishop for the knight on c6 and then locking in the black bishop with c5. In practice, this would imply that White has two pieces for the rook instead of being the exchange down. Of course, Black understood the threat and countered adequately with an exchange sacrifice. The continuation was in fact as follows: 14. c4 Bxf1 15. Nxf1 axb4 16. axb4 Rxa1 17. Bxa1 Nxb4, Black's adequate answer, 18. Bxe8 Qxe8 19. Bxe5 Qd7. The two combinations eventually led to a balanced position. Although both sides tried to improve their strengths, the game's status never moved much from a draw and this was agreed on move 69.

The game LEELA CHESS ZERO against THE BARON was very special, not for the opening and not for the combination on move 37 but for its endgame. To start at the midgame exit, we give as the point of departure 36... Rb6, Fig. 8c. The continuation was 37. e5 Bxf3 38. gxf3 Bc7 39. e6 f5 40. Qc2 g6 41. Ra1 Bb8 42. Ra8 Kh7 43. Qa4 Rb1+. This rook was predestined to play a pivotal role in the endgame. It was active up to move 138. Many times moves were repeated but the same position never occurred three times on the board so no draw could be claimed. THE BARON was aiming for the draw, LEELA CHESS ZERO was hoping for a win. Finally, THE BARON got its result - a draw.

The game BOOOT - SHREDDER was a correct game from both sides. First, they both knew the opening very well and then they applied the appropriate rules for handling the midgame positions with equal skill. Finally they exchanged a large amount of material in a computer way and both saw the end by perpetual check. Draw agreed.

In the game GRIDGINKGO - CHIRON, Black played the well-known Marshall variation of the Ruy Lopez opening. They followed the main ideas of this opening and White handled the position of a pawn up with care. This means that it limited Black's activities as was necessary and inevitably, the game ended in a draw.

Standings after Round 6: 1 KomOdo 41/2; 2 GRIDGINKGO 4; 3 SHREDDER 31/2; 4-5 CHIRON, JONNY 3;

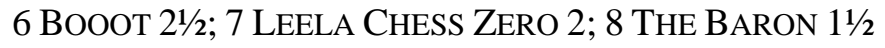

Round 7: CHIRON - KOMODO 1/2-1/2, SHREDDER - GRIDGINKGO 0-1, THE BARON - BOOOT 0-1, JONNY - LEELA CHESS ZERO 1-0 
In the last round, the pairing led to maximal excitement as the competitors in second and third places faced off against each other.
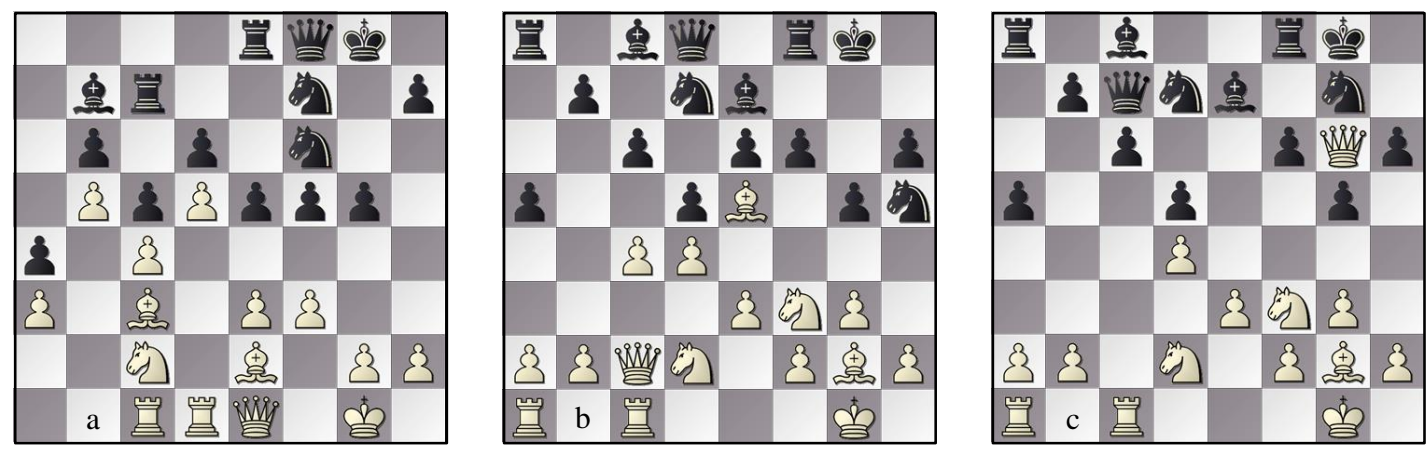

Fig. 9. R7: a) SHREDDER - GRIDGinKGo 26w; JoNNY - LEelA CHESs Zero (b) 16w and (c) 19w.

In the game CHIRON - KOMODO, Black defended itself using the Dutch opening, Leningrad variation. They played according to well-known examples in the textbook and the midgame followed the strategic lines that have been well known since the Botvinnik-Bronstein match of 1951. In the endgame, they managed to evoke some excitement in the evaluation function of human beings who replayed the game. For the computers all was clear, they remained in the neighbourhood of a draw and this was agreed after 69 moves.

SHREDDER had a last chance for a good qualification by winning the game against GRIDGINKGO. To judge from their manoeuvres immediately after the opening phase, the both started with ambitions for a win. The reconfiguring of the pieces was fascinating and spectators were eagerly waiting to see which of the two programs had followed the best strategy. When the clash finally came, by $\mathbf{2 5}$.... g5, it turned out that Black had its pieces well positioned. In Fig. 9a, 26. h4 was a mistake and from that moment Black had the initiative.

After some time, SHREDDER's king found himself reunited with the white queen directly beside him on h1. A superficial look at that position led all spectators to the conclusion 'this is a lost game' and so it was. White had no chance but the final position where Stefan Meyer-Kahlen resigned deserves some explanation. The obvious move 35. e4 is refuted by 35. ... Rxe4 36. fxe4 Nxe4 37. Re1 Nxc3 38. $\mathrm{Bg} 4 \mathrm{Ne} 5$.

THE BARON against BOOOT played a Queen's Gambit Accepted and followed the opening book up to move 13. For most players it will be a special line but the opponents looked like they knew this battlefield. Although THE BARON answered many difficult questions well, it was repeatedly facing new challenges. After two or three decisions which we could rate as second class, BOOOT clearly had the upper hand and this led to it winning the game - a result that put THE BARON in last place in this strong tournament. Even so, we can applaud an enormous performance if you take into account that its playing strength is assumed to be only a little better than that of the best human beings.

The game JONNY - LEELA CHESS ZERO was a game that attracted many spectators. It looked like a festival of sacrifices. The master of ceremony was Johannes Zwanzger who had the honour of executing the moves suggested by his program JONNY. It started by giving Black an opportunity to trap the black bishop. With a stop in between on e5 where Black played the mistaken move 15. ... f6, it landed on c7 where it was apparently taken for free by the black queen. That having been said, 
White had investigated this variation accurately: nowadays computers look far downstream - it is fascinating how powerful modern clusters are.

From the position of Fig. 9b, we follow the ideas presented above expressed in moves: 16. cxd5 exd5 17. Bc7 Qxc7. Now JONNY decided to penetrate the black position on the kingside with its queen: 18. Qg6+ Ng7. The question is how to proceed. For JONNY the answer was clear, see Fig. 9c. The threat on d5 was the essence of this move 19. Nxg5 fxg5 20. Bxd5+. Now Black was forced to give up his queen. 20... cxd5 21. Rxc7 Bd8 22. Rac1 Bxc7 23. Rxc7. In this position, Black has a rook and two pieces for the white queen. Materially it seems roughly equal but positionally the black fortress is no longer a fortress but rather a Swiss cheese with many holes. JONNY manoeuvred very well in the hectic position and brought in a full point, securing him the third place.

Standings after Round 7: 1-2 GRIDGINKGO, KomOdo 5; 3 JonNy 4; 4-6 BOOOT, CHIRON, SHREdDER 3112; 7 LEELA CHESS ZERO 2; 8 THE BARON 11/2

Thus, we moved to a play-off between GRIDGINKGO and KOMODO.

Table 2

The WCCC 2018 results

\begin{tabular}{|c|c|c|c|c|c|c|c|c|}
\hline id & Engine & r1 & r2 & r3 & r4 & r5 & r6 & r7 \\
\hline $\bar{G}$ & GRIDGINKGO & Tw1 & $\mathrm{Jb}^{1 / 2}$ & Lw1 & $\mathrm{Bb}^{1 / 2}$ & $\mathrm{Kw}^{1 / 2}$ & $\mathrm{Cw}^{1 / 2}$ & $\mathrm{Sb} 1$ \\
\hline K & KOMODO & Lb1 & $\mathrm{Sw}^{1 / 2}$ & $\mathrm{Bb} 1$ & Tw1 & $\mathrm{Gb}^{1 / 2}$ & $\mathrm{JW}^{1 / 2}$ & $\mathrm{Cb}^{1 / 2}$ \\
\hline $\mathbf{J}$ & JONNY & $\mathrm{Bb}^{1 / 2}$ & $\mathrm{Gw}^{1 / 2}$ & $\mathrm{Cb}^{1 / 2}$ & $\mathrm{Sw}^{1 / 2}$ & $\mathrm{~Tb}^{1} 1 / 2$ & $\mathrm{~Kb}^{1 / 2}$ & Lw1 \\
\hline B & BOOOT & $\mathrm{JWW}_{\mathrm{W} / 2}$ & $\mathrm{Lb}^{1 / 2}$ & Kw0 & $\mathrm{Gw}^{1 / 2}$ & $\mathrm{Cb}^{1} / 2$ & $\mathrm{Sw}^{1 / 2}$ & Tb1 \\
\hline $\mathrm{C}$ & CHIRON & $\mathrm{Sw}^{1 / 2}$ & $\mathrm{~Tb}^{1 / 2}$ & $\mathrm{JWW}^{1 / 2}$ & $\mathrm{Lb}^{1 / 2}$ & $\mathrm{Bw}^{1 / 2}$ & $\mathrm{~Gb}^{1 / 2}$ & $\mathrm{Kw}^{1 / 2}$ \\
\hline $\mathrm{S}$ & SHREDDER & $\mathrm{Cb}^{1 / 2}$ & $\mathrm{~Kb}^{1 / 2}$ & Tw1 & $\mathrm{Jb}^{1 / 2}$ & $\mathrm{Lw}^{1 / 2}$ & $\mathrm{Bb}^{1 / 2}$ & Gw0 \\
\hline $\mathrm{L}$ & EELA CHESS ZERO & Kw0 & $\mathrm{Bw}^{1 / 2}$ & $\mathrm{~Gb} 0$ & $\mathrm{Cw}^{1 / 2}$ & $\mathrm{Sb}^{1 / 2}$ & $\mathrm{Tw}^{1 / 2}$ & $\mathrm{Jb} 0$ \\
\hline $\mathrm{T}$ & THE BARON & Gb0 & $\mathrm{Cw}^{1 / 2}$ & $\mathrm{Sb} 0$ & $\mathrm{~Kb} 0$ & $\mathrm{Jw}^{1 / 2}$ & $\mathrm{Lb}^{1 / 2}$ & Bw0 \\
\hline
\end{tabular}

Tables 3 and 4

The WCCC 2018 cross-table and progress round by round

\begin{tabular}{|c|c|c|c|c|c|c|c|c|c|c|c|}
\hline Engine & $\mathbf{G}$ & $\mathbf{K}$ & $\mathbf{J}$ & $\mathbf{B}$ & $\mathbf{C}$ & $\mathbf{S}$ & $\mathbf{L}$ & $T$ & $\mathbf{W}$ & D & L Score \\
\hline GRIDGINKGO & & $1 / 2$ & $1 / 2$ & $1 / 2$ & $1 / 2$ & 1 & 1 & 1 & 3 & 4 & 5 \\
\hline KON & $1 / 2$ & & $1 / 2$ & 1 & $1 / 2$ & $1 / 2$ & 1 & 1 & 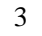 & 4 & 5 \\
\hline JON & $1 / 2$ & $1 / 2$ & & $1 / 2$ & $1 / 2$ & $1 / 2$ & 1 & $1 / 2$ & 1 & 6 & 4 \\
\hline $\mathrm{BOO}$ & $1 / 2$ & 0 & $1 / 2$ & & $1 / 2$ & $1 / 2$ & $1 / 2$ & 1 & 1 & 5 & $3 \frac{1}{2}$ \\
\hline CHIF & $1 / 2$ & $1 / 2$ & $1 / 2$ & $1 / 2$ & & $1 / 2$ & $1 / 2$ & $1 / 2$ & 0 & 7 & $0 \quad 3 \frac{1}{2}$ \\
\hline SHREDDER & 0 & $1 / 2$ & $1 / 2$ & $1 / 2$ & $1 / 2$ & & $1 / 2$ & 1 & 1 & 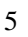 & $13 \frac{1}{1 / 2}$ \\
\hline EELA CHESS ZERO & 0 & 0 & 0 & $1 / 2$ & $1 / 2$ & $1 / 2$ & & $1 / 2$ & 0 & 4 & 2 \\
\hline THE BARON & 0 & 0 & $1 / 2$ & 0 & $1 / 2$ & 0 & $1 / 2$ & & 0 & & $1 \frac{1 / 2}{2}$ \\
\hline
\end{tabular}

\begin{tabular}{|c|c|}
\hline Engine & $\begin{array}{llllllll}\text { r1 } & \text { r2 } & \text { r3 } & \text { r4 } & \text { r5 } & \text { r6 } & \text { r7 } \\
\end{array}$ \\
\hline GRIDGINKGO & $\begin{array}{llllll}1 & 11 / 2 & 21 / 2 & 3 & 31 / 2 & 4\end{array}$ \\
\hline $\mathrm{KO}$ & $\begin{array}{lllllll}1 & 11 / 2 & 21 / 2 & 31 / 2 & 4 & 41 / 2 & 5\end{array}$ \\
\hline JO & $\begin{array}{llllll}1 / 2 & 1 & 11 / 2 & 2 & 2^{1 / 2} & 3\end{array}$ \\
\hline $\mathrm{BOO}$ & $\begin{array}{llllllll}1 / 2 & 1 & 1 & 11 / 2 & 2 & 2^{1} 1 / 2 & 31 / 2\end{array}$ \\
\hline CHIR & $\begin{array}{llllllll}1 / 2 & 1 & 11 / 2 & 2 & 21 / 2 & 3 & 3^{1 / 2}\end{array}$ \\
\hline SHREDDER & $\begin{array}{lllllll}1 / 2 & 1 & 2 & 2^{1 / 2} & 3 & 3^{1 / 2} & 31 / 2\end{array}$ \\
\hline ELA CH & $\begin{array}{llllll}0 & 1 / 2 & 1 / 2 & 1 & 11 / 2 & 2\end{array}$ \\
\hline THE BARON & $\begin{array}{lllllll}0 & 1 / 2 & 1 / 2 & 1 / 2 & 1 & 11 / 2 & 11 / 2\end{array}$ \\
\hline
\end{tabular}

The play-off. GRIDGINKGO - KOMODO 0-1, KOMODO - GRIDGINKGO 1/2-1/2

The play-off was scheduled as a series of three matches of two games, followed by an Armageddon. The first decisive match determined the result. The details were:

Match 1: each program had a time-budget of $30^{\prime}+15^{\prime \prime} / \mathrm{m}$,

Match 2: each program had a time-budget of $15^{\prime}+15^{\prime \prime} / \mathrm{m}$,

Armageddon: coin-toss determined colours, White $3^{\prime}+5^{\prime \prime} / \mathrm{m}$, Black $6^{\prime}+10^{\prime \prime} / \mathrm{m}$, a draw leaves Black as the victor. 
The first game, GRIDGINKGO - KOMODO, was fascinating and very educative for chess players at all levels. It was obvious that the level of the game was far above that of the strongest player in the world. The opening was played quietly and nicely, yet it already contained a series of rather adequate decisions. The most clearly weak decision observed was that White in the opening phase exchanged its black-coloured bishop for a black knight, leaving the pair of bishops to Black. It was considered by commentator and public as being of no significant importance, particularly as the position was closed and there was no fight on the two wings. But what is not on the board at one moment can appear on the board later. Specifically, the threat was now that the structure on the board might change and Black was particularly alert.
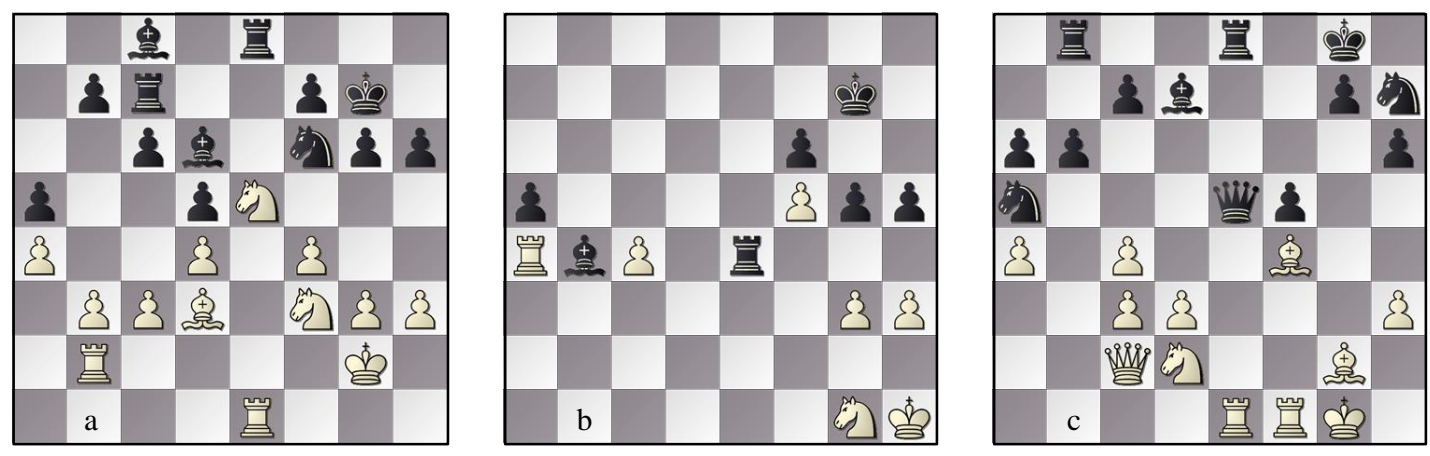

Fig. 10. Play-off: GRIDGinKGO - KomOdo a) 31 b and b) 59w; c) KoMODO - GRIDGinKGO 22b.

Believe it or not, KoMODO knew what other human players and spectators did not know. KomODO knew through its evaluation function that Black could expect to have a clear advantage soon, namely at the moment that it could open the position and split the board into two fighting areas. Indeed, Black took its opportunity as in Fig. 10a with 31.... c5. This move did indeed split the position into two parts and gave the pair of bishops the advantage over White's bishop and knight. For the computers, the remainder was a matter of technique. To show you what type of position Black had in mind we reproduce in Fig. 10b the position after 58. ... Bb4 where the power of the black bishop is shown in full detail. Black won on move 73.

The second play-off game was to be decisive. KOMODO's task was to achieve at least a draw. Still, computers remain computers and therefore we saw KOMODO going for a second win. Of course, GRIDGINKGO had the same in mind. The result was a very fascinating game. In fact, this game was more exciting than the first game. After 22. Bf4, as in Fig. 10c, it looked like Komodo had the lead.

The material balance became difficult to evaluate as positional considerations rather than material per se played the main role. The forcing power of White's moves rendered material balance a secondary consideration. Black's counter-play was well thought out and ingenious. Then the question arose as to whether the position was still favourable for White. The play took place at a very high level and suddenly there was such a difficult position that no player could make any progress. Of course, the position was 'milked' almost to the very end but finally both sides understood over a handshake that a draw was unavoidable.

KomODO was therefore declared the winner of the 24th World Computer Chess Championship. Our congratulations go particularly to the KOMODO team, as well as to GRIDGINKGO's team of authors who received a prize of CHESSBASE14 donated by Chessbase. All participants demonstrated fine play 
as evidenced by Harry Schussler's excellent commentary and an examination of the games (Krabbenbos et al., 2018) will be most rewarding.

\section{REFERENCES}

CPW (2018). https://tinyurl.com/icga046. Biographies of programs, authors and operators.

Krabbenbos, J., van den Herik, H.J. and Haworth, G.M ${ }^{\mathrm{c} C}$. (2018). WCCC 2018: the $24^{\text {th }}$ World Computer Chess Championship. ICGA Journal, 40(3), 182-193. doi: 10.3233/ICG-190080. Pgn file available from http://centaur.reading.ac.uk/78436/.

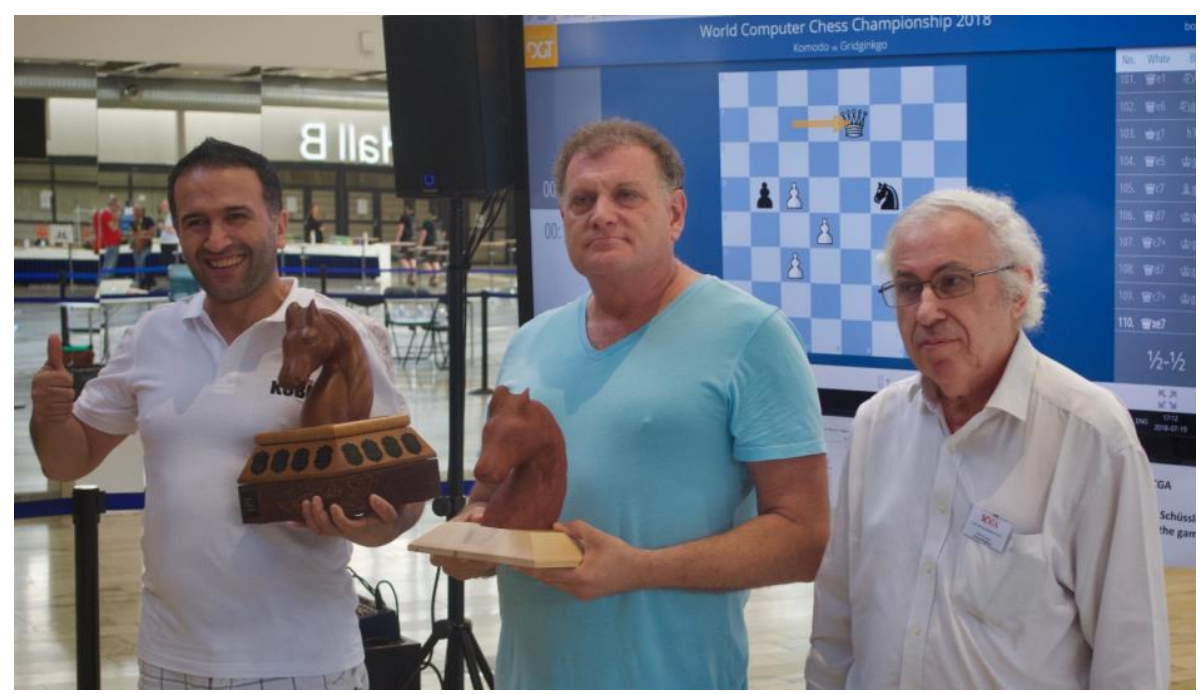

Fig. 11. Winner Komodo's team members Erdogan Günes (1) and Mark Lefler with ICGA President David Levy (r).

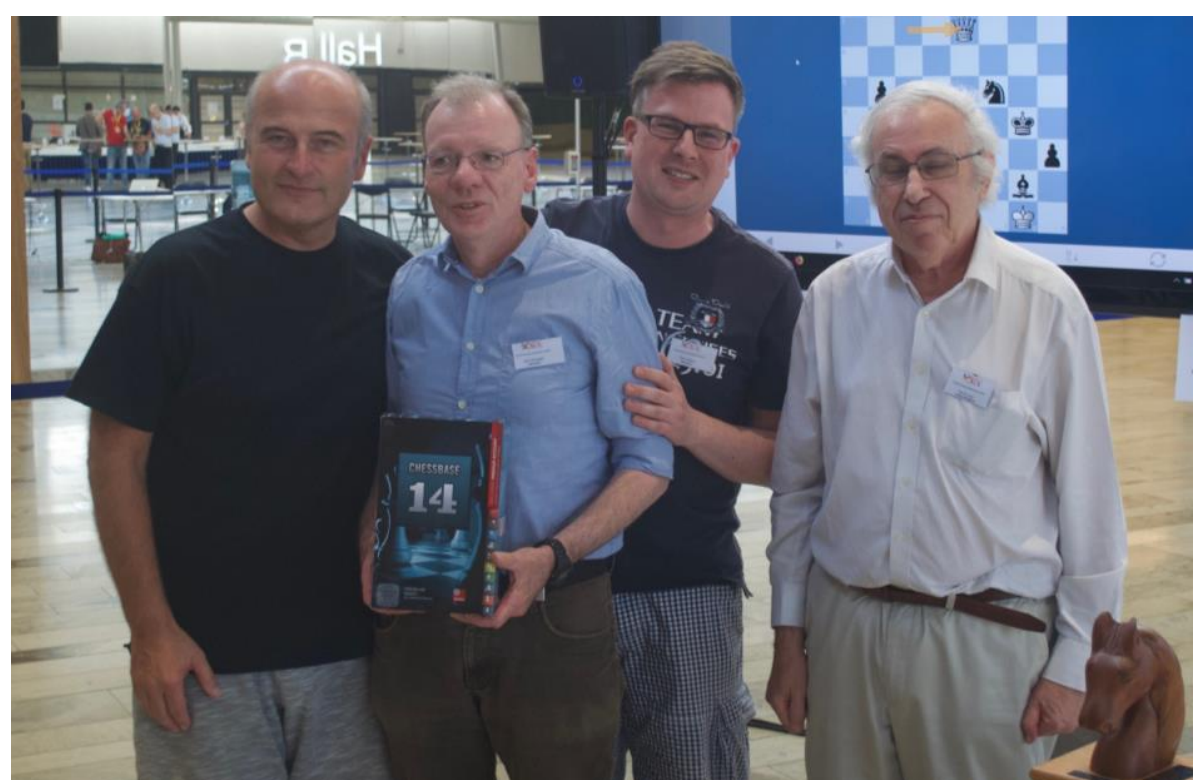

Fig. 12. Runner-up GRIDGINKGO’s team members Wolfgang Zugrav, Kai Himstedt and Timo Haupt, and David Levy. 